\title{
Papel de las adAPTACIONES EPIGENÉTICAS EN EL RIESGo DE ENFERMEDAdES CARDIOVASCULARES EN LA POBLACIÓN LATINOAMERICANA
}

\author{
Patricio López-Jaramillo ${ }^{1,2 *}$, Daniel Cohen ${ }^{3}$, Diego Gómez-Arbeláez ${ }^{2}$, Carlos Velandia ${ }^{1,2}$ \\ Arístides Sotomayor-Rubio ${ }^{4}$ Katherine Rincón-Romero², José López-López ${ }^{4}$ \\ ${ }^{1}$ Dirección de Investigaciones, Desarrollo e Innovación Tecnológica, Fundación Oftalmológica de Santander- \\ Clínica Carlos Ardila Lulle (FOSCAL). 'Dirección de Investigaciones, Facultad de Medicina, Universidad de Santander (UDES). \\ ${ }^{3}$ Faculty of Life Sciences, London Metropolitan University, London UK. \\ ${ }^{4}$ Facultad de Medicina, Universidad Autónoma de Bucaramanga (UNAB)
}

\begin{abstract}
Resumen
Las enfermedades cardio-metabólicas (obesidad, diabetes mellitus tipo 2, infarto agudo de miocardio y accidente cerebro-vascular) son al momento el problema de salud pública más relevante en Colombia. La rápida urbanización experimentada por el país en los últimos 50 años, así como los cambios en los hábitos nutricionales y en la actividad física asociada a la urbanización han determinado que en el proceso de adaptación biológica de la población colombiana a este rápido proceso medioambiental, se sobre expresen por vía epigenética, substancias pro inflamatorias y aquellas que llevan a resistencia a la insulina, mecanismo que confiere una mayor susceptibilidad para que nuestra población desarrolle las enfermedades cardio-metabólicas. Este proceso es especialmente grave en los niños escolares de nuestras ciudades. De frente a esta situación, en el presente artículo revisamos una serie de evidencias que nos llevan a proponer que el ejercicio, especialmente el de fuerza puede, vía epigenética, determinar un mayor desarrollo de masa muscular y la expresión de sustancias producidas en el músculo que tienen propiedades anti inflamatorias y que mejoran la sensibilidad a la insulina, por lo cual se contraponen al estado de resistencia a la insulina y a la inflamación de bajo grado, producto de la obesidad especialmente abdominal.
\end{abstract}

Palabras clave: actividad física, epigenética, enfermedades cardiovasculares, diabetes mellitus, obesidad

\section{Role OF THE EPIGENETIC ADAPTATIONS IN THE RISK OF CARDIOVASCULAR DISEASE IN LATIN AMERICAN POPULATIONS}

\begin{abstract}
Cardio metabolic disorders (obesity, type-2 diabetes, myocardial infarction and stroke) are nowadays the most important health problem in Colombia. The rapid process of urbanization experimented by the Colombian population in the last 50 years, together with the changes in nutrition and physical activity associated to urbanization, has been related to an epigenetic adaptation in which the genes that regulate the mechanisms of insulin resistance and low degree inflammation have been over express. This mechanism is generating a high sensibility in our population to develop cardio metabolic disorders. The changes in physical activity and in nutrition behavior are especially important in school-age children. In this article we review the evidences that supports the proposal that an increase in physical activity in school-age children, mainly strength activities will develop, through epigenetic mechanisms, an increase in muscular mass and the development of biological substances in the muscles that have the ability to improve insulin sensibility; they also have anti-inflammatory effects. These actions of the increased muscular mass will improve insulin resistance and low degree inflammation produced by abdominal obesity.
\end{abstract}

Key words: physical activity, epigenetics, cardiovascular diseases, diabetes mellitus, obesity

Recibido: Mayo 13 de $2011 \quad$ Aceptado: Junio 15 de 2011

* Correspondencia: Patricio López-Jaramillo MD PhD FACP jplopezj@gmail.com; investigaciones@foscal.com.co. Dirección postal: Fundación Oftalmológica de Santander - Clínica Carlos Ardila Lulle (FOSCAL), Torre Milton Salazar, Primer Piso. Entrada Oftalmología y Farmacia, Calle 155 A N. 23-09, Urbanización El Bosque, Floridablanca - Santander, Colombia. Phone: 57-3153068939, 57 7-6384160/6386000 Ext 2509/2510, Fax: 57-7-63881084 


\title{
Papel das ADAPTAÇões EPIGENÉTICAS NO RISCO DAS DOENÇAS CARDIOVASCULARES NA POPULAÇÃO LATINO-AMERICANA
}

\begin{abstract}
Resumo
As doenças cardiometabólicas (obesidade, diabetes mellitus tipo 2, infarto agudo do miocárdio e acidente vascular cerebral) são no momento o problema de saúde pública mais relevantes na Colômbia. A rápida urbanização experimentada pelo país nos últimos 50 anos, bem como mudança nos hábitos alimentares e atividade física associada com a urbanização têm determinado que no processo de adaptação biológica da população colombiana a este rápido processo ambiental, fique em sobre-expressão pela via epigenética substâncias pró-inflamatórias e aqueles que levam à resistência à insulina, um mecanismo que confere aumento da susceptibilidade para que a nossa população desenvolva as doenças cardio-metabólicas. Este processo é particularmente grave em crianças em idade escolar de nossas cidades. Perante esta situação, este artigo analisa uma série de evidências que nos levam a propor que o exercício, especialmente de força pode, via epigenética determinar um maior desenvolvimento de massa muscular e expressão de substâncias produzidas no músculo que têm propriedades anti-inflamatórias e melhoram a sensibilidade à insulina, que se opõe o estado de resistência à insulina e ainflamação de baixo grau produto da obesidade, especialmente a abdominal.
\end{abstract}

Palavras-chave: atividade física, epigenética, doenças cardiovasculares, diabetes mellitus, obesidade

\section{Introducción}

La obesidad es un problema de salud pública que esta afectando a la población mundial, y que se relaciona con la epidemia de enfermedades cardio-metabólicas (ECM) que se observa actualmente en Latinoamérica (1). Existen notables diferencias regionales en el comportamiento de la prevalencia de las enfermedades cardiovasculares (ECV) y de la diabetes mellitus tipo 2 (DM2). Así, en los últimos años la prevalencia de ECM se ha incrementando de forma exponencial en los países del tercer mundo (1), mientras en los países desarrollados, a pesar del incremento en las tasas de obesidad, la prevalencia de ECV va disminuyendo (2). Adicionalmente, varios estudios han mostrado que aproximadamente el $40 \%$ de la población obesa en los países desarrollados no presentan resistencia a la insulina (RI) ni aumento en los factores de riesgo cardiovascular clásicos por lo que se consideran individuos con un fenotipo metabólico saludable (3-5). De otro lado se ha descrito que sujetos hispanos viviendo en Estados Unidos (EUA) tienen un riesgo incrementado de desarrollar resistencia a la insulina, obesidad, inflamación de bajo grado, DM2 y ECV $(6,7)$. El estudio INTERHEART Latinoamérica (8) y el estudio INTERSTROKE (9), dos grandes estudios epidemiológicos internacionales, demostraron que en los países latinoamericanos la obesidad abdominal es el factor de riesgo con el mayor valor de riesgo poblacional atribuible para la presencia de un primer infarto agudo de miocardio (IAM) y un primer accidente cerebrovascular isquémico o hemorrágico.
A pesar de que es bien conocido el rol de la obesidad en el aumento del riesgo de ECM, los mecanismos etiológicos subyacentes no son bien entendidos. En niños como en adultos, el exceso de adiposidad es una determinante constante de la inflamación crónica de bajo grado que tiene un papel protagónico en el desarrollo de ECM (10). El tejido adiposo es un órgano secretor activo que libera a la circulación factores del complemento y citoquinas proinflamatorias, particularmente interleuquina 6 (IL-6) y factor de necrosis tumoral alfa (TNF-a) los cuales a su vez, estimulan la producción de proteína c-reactiva (PCR), un marcador inflamatorio con alta sensibilidad para demostrar la presencia de inflamación sistémica $(11,12)$.

Diferentes estudios han evaluado las concentraciones de PCR en niños escolares de países desarrollados, mostrando que las concentraciones séricas de PCR están correlacionadas con el IMC, y que en esta asociación se encuentran diferencias significativas entre diferentes etnias. Específicamente se observó concentraciones más altas de PCR en niños mexicano-americanos comparados con caucásicos $(10,13)$. En Colombia se ha demostrado una relación positiva significativa entre IMC y PCR, y consecuentemente valores más altos de PCR en niños y niñas del tercil más alto en la distribución del IMC (14). Es importante destacar que las concentraciones de PCR encontradas en nuestros niños sin sobrepeso ni obesidad que se encontraban en el segundo tercil de peso, fueron tan altas como las reportadas en estudios con niños caucásicos de similar edad con obesidad $(15,16)$. 
En perspectiva de explicar este fenómeno se ha propuesto que los habitantes de países en vía de desarrollo tienen una mayor predisposición a desarrollar inflamación de bajo grado, RI, y ECM a grados más bajos de sobrepeso y obesidad (17) y que esto puede ser debido a diferencias epigenéticas y adipogénicas de esta población comparada con la población de países desarrollados tales como EUA (18). La propuesta de una mayor predisposición de los individuos del tercer mundo para desarrollar RI e inflamación de bajo grado ha sido estudiada por nuestro grupoen adultos colombianos, los cuales son más propensos a desarrollar resistencia a la insulina, inflamación de bajo grado y ECV con perímetros abdominales menores cuando se comparan con población caucásica (19-25)

Los cambios fisiopatológicos en los individuos con ECM empiezan en etapas tempranas, incluso antes de la presencia de cualquier sintomatología. Estudios epidemiológicos y experimentales de intervención en la dieta, han sugerido que el desbalance en la nutrición materna y alteraciones metabólicas en la vida intrauterina o en los primeros años de vida pueden tener un efecto persistente en la salud y bienestar en el adulto e incluso podría ser transmitido a las siguientes generaciones $(26,27)$. Los seres humanos no estuvieron biológicamente preparados para la transición acelerada hacia estilos de vida modernos, especialmente a los rápidos cambios que las poblaciones de los países en vía de desarrollo han experimentado los últimos años. Por siglos estas poblaciones (las de países en vía de desarrollo) estuvieron expuestas a escasez de comida y a grandes demandas de actividad física. Recientemente experimentaron un abrupto cambio y actualmente están expuestas a un sedentarismo tanto en sus actividades cotidianas de trabajo como también, y especialmente, en las actividades recreativas. En contraste, las poblaciones de países desarrollados tuvieron una transición mucho más lenta, con mayor tiempo de exposición al estilo de vida moderno y por lo tanto han desarrollado respuestas adaptativas a los excesos del estilo de vida moderno $(26,27)$.

\section{Tejido adiposo, músculo y citoquinas}

Existe evidencia de la existencia de un sistema de información cruzada entre el músculo esquelético y el tejido adiposo con capacidad endocrina (28). De particular interés son los reportes que hacen relación a la interacción entre la masa grasa, la masa muscular y la producción de adipoquinas. Las citoquinas derivadas del músculo (mioquinas), en particular las producidas en las fibras musculares tipo II (29-31), promueven un ambiente antiinflamatorio y antiaterogénico a través de efectos sistémicos y/o específicos en la grasa visceral y en otros tejidos (32). En los seres humanos, por ejemplo, los niveles plasmáticos de la mioquina antiinflamatoria IL-15, están inversamente relacionados con la cantidad de masa grasa existente en el tronco. Experimentalmente se ha observado que la sobre expresión de esta mioquina reduce la masa grasa visceral en ratones (33). En contraste, la citoquina proinflamatoria TNF- $\alpha$ inhibe la síntesis proteica muscular, por lo tanto se asociada con menor fuerza transversal (34), y predice la pérdida de fuerza longitudinal (35). En población no hispánica existen evidencias de que las citoquinas inflamatorias se asocian inversamente con la fuerza muscular (36) y el estado aeróbico (37) y con la cantidad de masa corporal magra (38). Si bien los datos de referencia en los niños hispánicos son limitados, los pocos datos existentes sugieren que estas tres mediciones relacionadas con la masa/calidad del músculo pueden ser menores en la población hispánica. Así, la fuerza del segmento superior determinado por dinamometría es menor en niños colombianos (39) comparado a los valores de referencia de UK (40), así como también en adultos brasileños cuando se comparan con los valores de referencia de Europa o de EUA (41). La Encuesta de Salud y Nutrición de los Estados Unidos (NHANES: National Health and Nutrition Examination Survey) demostró la existencia de una menor masa muscular en adultos mexicano-americanos en relación los observados en caucásicos americanos (42). También se ha reportado una menor capacidad aeróbica en niños hispano-americanos, independientemente de su composición corporal (43).

Una importante consideración para interpretar los resultados anteriores es la que hace relación a la alta prevalencia de desnutrición fetal y en la niñez que se observa entre los niños colombianos. Es bien conocido que la desnutrición intrauterina y durante la infancia produce alteraciones epigenéticas en una variedad de células y tejidos (27), adaptaciones epigenéticas que incrementan el riesgo de anormalidades metabólicas y enfermedades en la vida adulta (44). Una de estas adaptaciones que se presenta durante la etapa de plasticidad del desarrollo fetal es la que determina una menor masa corporal magra (45) y una menor fuerza muscular (46), las que se presentan en la vida adulta de individuos que presentaron un bajo peso al nacer para la edad gestacional. La correlación entre bajo peso al nacer, menor masa corporal magra y disminuida fuerza muscular es estadísticamente muy robusta, al punto de que estos parámetros son considerados como marcadores de desnutrición fetal. Varios trabajos han demostrado que en los individuos con antecedentes de bajo peso al nacer 
es común la presencia de deficiencias en las proteínas de señalización intracelular de la insulina en el tejido muscular y adiposo, en las proteínas de señalización anabólica del músculo, además de alteraciones en la composición de la fibra muscular (47-50). Interesantemente, se ha observado que el elevado riesgo de desarrollar síndrome metabólico que presentan los individuos con bajo peso al nacer, desaparece en aquellos individuos que presentan un buen estado físico aeróbico o que realizan niveles significativos de actividad muscular vigorosa (51).

Por lo tanto, además de la cantidad de masa grasa y su distribución, la cantidad y calidad de la masa muscular, así como el nivel de función muscular, podrían contribuir a la elevación de citoquinas inflamatorias circulantes en el torrente sanguíneo $(14,52)$, situación que esta en concordancia con la propuesta de la mayor susceptibilidad para desarrollar ECV que se observa en la población latinoamericana (17). En soporte de nuestra propuesta, un reciente trabajo realizado en USA, demostró la existencia de una asociación inversa entre el porcentaje de masa corporal magra y el riesgo de presentar enfermedad coronaria en adultos jóvenes hispánicos, asociación que no se observó en los blancos o negros no hispánicos (53).

Con base en los resultados que demuestran que la contribución de la masa grasa abdominal al riesgo poblacional atribuible de ECV es mayor en la población latinoamericana que en la europea o norteamericana $(8,9)$, y vistos los argumentos que demuestran un efecto endocrino compensador de la masa muscular, proponemos que la interacción entre estos dos tejidos es fundamental para determinar el riesgo de presentar ECM en nuestra población.

La relación entre fuerza muscular y marcadores inflamatorios no ha sido evaluada en la población Latino Americana, pero si existen datos prometedores de que los niños y adultos hispánicos con sobrepeso o diabetes mejoran la sensibilidad a la insulina después de la práctica de ejercicio $(54,55)$. Además, en la población colombiana hemos demostrado que el ejercicio mejora la función endotelial en mujeres embarazadas (55) y que la administración de intervenciones nutricionales como extractos de ajo añejado a pacientes con síndrome metabólico incrementa los niveles de adiponectina (56). En niños, la fuerza del segmento superior determinado por dinamometría es una medida fácil de obtener, segura y valiosa para medir fuerza muscular $(57,58)$, la cual se relaciona fuertemente con el índice de fuerza superior e inferior de la masa muscular (59) y la capacidad aeróbica (60). Por lo tanto, en este momento con financiamiento de la Alcaldía de Bucaramanga y de la Fundación MAPFRE de España y con la colaboración de la Universidad Metropolitana de Londres y el Hospital Nacional de Niños de Washington D.C., nos encontramos estudiando las variaciones epigenómicas del tejido graso en relación al IMC, a los niveles de adipocitoquinas y a las interacciones con la medición de masa y función muscular en escolares de Bucaramanga, en comparación con escolares de ascendencia hispana que viven en Washington y con niños caucásicos de la misma ciudad.

\section{Aspectos genéticos y epigenéticos de la obesidad}

Se cree que la obesidad y las co-morbilidades asociadas a la misma tales como las ECM son el resultado de una compleja interacción entre el medio ambiente y la genética. Estudios realizados en gemelos y entre familiares de obesos han reportado la existencia de una herencia de la obesidad, encontrando que la contribución genética es de alrededor del 50\% (61). Se ha generado una gran cantidad de resultados con la utilización de estudios de asociación del genoma humano o GWAS (genome wide association studies), en la población con obesidad y peso normal, que han llevado a la identificación de decenas de loci genéticos que predisponen a la presencia de obesidad $(62,63)$. Sin embargo, los nuevos datos han llevado a una enorme contradicción pues se observa que la sumatoria del efecto de más de diez de los mas significantes loci genéticos explican solamente alrededor de 1-2\% de la variación poblacional de la obesidad. En otras palabras, estos estudios soportan la visión de que hay una significante "falta de herencia" en la aparición de la obesidad (64).

Es posible que existan algunos genotipos muy raros que no son detectados por GWAS, y que acumulativamente podrían compensar parte de esta "falta de herencia". Es así como recientemente se identificó una rara microdeleción de un locus genético (65). Sin embargo, un mecanismo alternativo que está ganando un acelerado apoyo es el de la existencia de alteraciones epigenómicas tales como los patrones de metilación de genes que pueden ser modificados por el ambiente y que en algunos casos, pueden heredarse. De hecho, en este momento existe gran cantidad de evidencias que demuestran que las alteraciones epigenéticas del genoma son un factor de riesgo importante para la obesidad, y que tal vez explican la epidemia de obesidad observada especialmente en países en transición económica $(66,67)$.

Por lo tanto, es oportuno e importante entender mejor el rol de la epigenética en la obesidad. El análisis epigenético 
de todo el genoma promete aumentar el conocimiento en términos de diferencias entre los depósitos de grasa (visceral vs. subcutánea), riesgo relativo entre individuos en ambientes idénticos, y la interacción entre el medio ambiente y los genes de un individuo. Esto a su vez debería conducir a enfoques terapéuticos más racionales y selectivos, quizá incluyendo intervenciones con medicina personalizada.

\section{Referencias}

1. Murray CJ, Lopez AD. Global mortality, disability, and the contribution of risk factors: Global Burden of Disease Study. Lancet. 1997;349:1436-42.

2. Ford ES, Ajani UA, Croft J B et al. Explaining the decrease in U.S. deaths from coronary disease, 1980-2000. N Engl J Med. 2007;356:2388-98.

3. Brochu M, Tchernof A, Dionne IJ, et al. What are the physical characteristics associated with a normal metabolic profile despite a high level of obesity in postmenopausal women? J Clin Endocrinol Metab. 2001;86:1020-25.

4. Ferrannini E, Natali A, Bell P, Cavallo-Perin P, Lalic N, Mingrone G. Insulin resistance and hypersecretion in obesity. European Group for the Study of Insulin Resistance (EGIR). J Clin Invest. 1997;100:1166-73.

5. Karelis AD, Brochu M, Rabasa-Lhoret R. Can we identify metabolically healthy but obese individuals (MHO)? Diabetes Metab. 2004;30:569-72.

6. Ogden CL, Flegal KM, Carroll MD, Johnson CL. Prevalence and trends in overweight among US children and adolescents, 19992000. JAMA. 2002;288:1728-32.

7. Okosun I S, Liao Y, Rotimi CN, Prewitt TE, Cooper RS. Abdominal adiposity and clustering of multiple metabolic syndrome in White, Black and Hispanic americans. Ann Epidemiol. 2000;10:263-70.

8. Lanas F, Avezum A, Bautista LE, et al. Risk factors for acute myocardial infarction in Latin America: the INTERHEART Latin American study. Circulation. 2007;115:1067-74.

9. O'Donnell M J, Xavier D, Liu L et al. Risk factors for ischaemic and intracerebral haemorrhagic stroke in 22 countries (the INTERSTROKE study): A case-control study. Lancet. 2010;376:11223.

10. Ford ES. C-reactive protein concentration and cardiovascular disease risk factors in children: findings from the National Health and Nutrition Examination Survey 1999-2000. Circulation. 2003;108:1053-58.

11. Baumann H, Gauldie J. Regulation of hepatic acute phase plasma protein genes by hepatocyte stimulating factors and other mediators of inflammation. Mol Biol Med. 1990;7:147-59.

12. Wellen KE, Hotamisligil GS. Obesity-induced inflammatory changes in adipose tissue. J Clin Invest. 2003;112:1785-1788.

13. Cook DG, Mendall MA, Whincup P H et al. C-reactive protein concentration in children: relationship to adiposity and other cardiovascular risk factors. Atherosclerosis. 2000;149:139-50.
14. López-Jaramillo P, Herrera E, García RG, Camacho PA, Castillo VR. Inter-relationships between body mass index, C-reactive protein and blood pressure in a Hispanic pediatric population. Am J Hypertens. 2008;21:527-32.

15. Aeberli I, Molinari L, Spinas G, Lehmann R, l'Allemand D, Zimmermann MB. Dietary intakes of fat and antioxidant vitamins are predictors of subclinical inflammation in overweight Swiss children. Am J Clin Nutr. 2006;84:748-55.

16. Weiss R, Dziura J, Burgert T S et al. Obesity and the metabolic syndrome in children and adolescents. N Engl J Med. 2004;350:236274.

17. López-Jaramillo P. Defining the research priorities to fight the burden of cardiovascular diseases in Latin America. J Hypertens. 2008;26:1886-89.

18. López-Jaramillo P, Silva SY, Rodriguez-Salamanca N, Duran A, Mosquera W, Castillo V. Are nutrition-induced epigenetic changes the link between socioeconomic pathology and cardiovascular diseases? Am J Ther. 2008;15:362-72.

19. García RG, Cifuentes AE, Caballero RS, Sanchez L, Lopez-Jaramillo P. A proposal for an appropriate central obesity diagnosis in Latin American population. Int J Cardiol. 2006;110:263-64.

20. García RG, Pérez M, Maas R, Schwedhelm E, Boger RH, LopezJaramillo P. Plasma concentrations of asymmetric dimethylarginine (ADMA) in metabolic syndrome. Int J Cardiol. 2007;122:176-8.

21. García RG, Rincón MY, Arenas W D et al. Hyperinsulinemia is a predictor of new cardiovascular events in Colombian patients with a first myocardial infarction. Int J Cardiol. 2011;148:85-90.

22. López-Jaramillo P, Rueda-Clausen CF, Silva FA. The utility of different definitions of metabolic syndrome in Andean population. Int J Cardiol. 2007;116:421-2.

23. Pérez M, Casas J P, Cubillos-Garzon L A et al. Using waist circumference as a screening tool to identify Colombian subjects at cardiovascular risk. Eur J Cardiovasc Prev Rehabil. 2003;10:328-35.

24. Rueda-Clausen CF, Lahera V, Calderon J, et al. The presence of abdominal obesity is associated with changes in vascular function independently of other cardiovascular risk factors. Int J Cardiol. 139:32-41.

25. Rueda-Clausen CF, Lopez-Jaramillo $P$, Luengas $C$, del Pilar Oubina $\mathrm{M}$, Cachofeiro V, Lahera V. Inflammation but not endothelial dysfunction is associated with the severity of coronary artery disease in dyslipidemic subjects. Mediators Inflamm. 2009;2009:ID 469169.

26. Gallou-Kabani C, Junien C. Nutritional epigenomics of metabolic syndrome: new perspective against the epidemic. Diabetes. 2005;54:1899-906.

27. Lopez-Jaramillo P. Cardiometabolic disease in Latin America: the role of fetal programming in response to maternal malnutrition. Rev Esp Cardiol. 2009;62:670-76

28. Argiles JM, Lopez-Soriano J, Almendro V, Busquets S, LopezSoriano FJ. Cross-talk between skeletal muscle and adipose tissue: a link with obesity? Med Res Rev. 2005;25:49-65.

29. Hiscock N, Chan MH, Bisucci T, Darby IA, Febbraio MA. Skeletal myocytes are a source of interleukin-6 mRNA expression and protein release during contraction: Evidence of fiber type specificity. Faseb J. 2004;18:992-4. 
30. Nielsen AR, Mounier R, Plomgaard P, et al. Expression of interleukin-15 in human skeletal muscle: effect of exercise and muscle fibre type composition. J Physiol. 2007;584:305-12.

31. Walsh K. Adipokines, myokines and cardiovascular disease. Circ J. 2009;73:13-8.

32. Brandt $\mathrm{C}$, Pedersen BK. The role of exercise-induced myokines in muscle homeostasis and the defense against chronic diseases. J Biomed Biotechnol. 2010;2010:ID 520258.

33. Nielsen A R, Hojman P, Erikstrup C et al. Association between interleukin-15 and obesity: interleukin-15 as a potential regulator of fat mass. J Clin Endocrinol Metab. 2008;93:4486-93.

34. Visser M, Pahor M, Taaffe D R et al. Relationship of interleukin-6 and tumor necrosis factor-alpha with muscle mass and muscle strength in elderly men and women: the Health $\mathrm{ABC}$ Study. J Gerontol A Biol Sci Med Sci. 2002;57:326-32.

35. Schaap LA, Pluijm SM, Deeg D J et al. Higher inflammatory marker levels in older persons: associations with 5-year change in muscle mass and muscle strength. J Gerontol A Biol Sci Med Sci. 2009;64:1183-89.

36. Ruiz JR, Sui X, Lobelo $F$ et al. Association between muscular strength and mortality in men: prospective cohort study. BMJ. 2008;337:439.

37. Ruiz JR, Ortega F B, Warnberg J et al. Inflammatory proteins and muscle strength in adolescents: the Avena study. Arch Pediatr Adolesc Med. 2008;162:462-68.

38. Donges C E, Duffield R, Drinkwater EJ. Effects of resistance or aerobic exercise training on interleukin-6, C-reactive protein, and body composition. Med Sci Sports Exerc. 2010;42:304-13.

39. Ramos-Bermudez S, Alonso-Alzate G, Salazar D. Evaluación antropométrica y motriz condicional de niños y adolescentes. 1 $^{\mathrm{a}}$ ed. Manizales, Colombia: Editorial Universidad de Caldas; 2007.

40. Cohen D, Voss C, taylor M, Delextrat A, Ogunleye A, Sandercock G. Ten-Year secular changes in muscular fitness in English children. Acta Paediatr. 2011;100:175-77.

41. Schlussel MM, dos Anjos LA, de Vasconcellos MT, Kac G. Reference values of handgrip dynamometry of healthy adults: a population-based study. Clin Nutr. 2008;27:601-7.

42. Chumlea WC, Guo SS, Kuczmarski R J et al. Body composition estimates from NHANES III bioelectrical impedance data. Int J Obes Relat Metab Disord. 2002;26:1596-609.

43. Shaibi GQ, Ball GD, Goran MI. Aerobic fitness among Caucasian, African-American, and Latino youth. Ethn Dis. Winter. 2006;16:120-5.

44. Hales CN, Barker DJ. The thrifty phenotype hypothesis. Br Med Bull. 2001;60:5-20.

45. Wells JC, Chomtho S, Fewtrell MS. Programming of body composition by early growth and nutrition. Proc Nutr Soc. 2007;66:423-34.

46. Sayer AA, Cooper C. Fetal programming of body composition and musculo skeletal development. Early Hum Dev. 2005;81:735-44.

47. Ozanne SE, Jensen CB, Tingey KJ, Storgaard H, Madsbad S, Vaag AA. Low birthweight is associated with specific changes in muscle insulin-signalling protein expression. Diabetologia. 2005;48:547-52.

48. Ozanne SE, Jensen CB, Tingey K J et al. Decreased protein levels of key insulin signaling molecules in adipose tissue from young men with a low birth weight: potential link to increased risk of diabetes? Diabetologia. 2006;49:2993-9.
49. Jensen CB, Martin-Gronert MS, Storgaard H, Madsbad S, Vaag A, Ozanne SE. Altered PI3-kinase/Akt signalling in skeletal muscle of young men with low birth weight. PLoS One. 2008;3:3738.

50. Jensen CB, Storgaard H, Madsbad S, Richter EA, Vaag AA. Altered skeletal muscle fiber composition and size precede whole-body insulin resistance in young men with low birth weight. J Clin Endocrinol Metab. 2007;92:1530-4.

51. Laaksonen DE, Lakka H M, Lynch J et al. Cardio respiratory fitness and vigorous leisure-time physical activity modify the association of small size at birth with the metabolic syndrome. Diabetes Care. 2003;26:2156-64.

52. Nazmi A, Victora CG. Socioeconomic and racial/ethnic differentials of C-reactive protein levels: a systematic review of populationbased studies. BMC Public Health. 2007;7:212.

53. Koutoubi S, Huffman FG. Body composition assessment and coronary heart disease risk factors among college students of three ethnic groups. J Natl Med Assoc. 2005;97:784-91.

54. Brooks N, Layne JE, Gordon PL, Roubenoff R, Nelson ME, Castaneda-Sceppa C. Strength training improves muscle quality and insulin sensitivity in Hispanic older adults with type 2 diabetes. Int J Med Sci. 2007;4:19-27.

55. Ramírez-Vélez R, Aguilar de Plata AC, Escudero MM, Echeverry I, Ortega JG, Salazar B, Rey JJ, Hormiga C, López-Jaramillo P. Influence of regular aerobic exercise on endothelium-dependent vasodilation and cardio respiratory fitness in pregnant women. J Obstet Gynecol Res. 2011;37:1601

56. López-Jaramillo P, Oubiña P, Valero S, Ballesteros S, Lahera V, Rodriguez Y, Garcia R, Gomez-Arbelaez D. Aged Garlic Extract increases adiponectin levels in subjects with metabolic syndrome. J. Diab 2011;3:51.

57. Shaibi GQ, Cruz ML, Ball G D et al. Effects of resistance training on insulin sensitivity in overweight Latino adolescent males. Med Sci Sports Exerc. 2006;38:1208-15.

58. Sherriff A, Wright CM, Reilly JJ, McColl J, Ness A, Emmett P. Age- and sex-standardised lean and fat indices derived from bioelectrical impedance analysis for ages 7-11 years: functional associations with cardio-respiratory fitness and grip strength. $\mathrm{Br} J$ Nutr. 2009;101:1753-1760.

59. Ortega FB, Artero EG, Ruiz J R et al. Reliability of health-related physical fitness tests in European adolescents. The HELENA Study. Int J Obes. 2008;32:49-57.

60. Milliken LA, Faigenbaum AD, Loud RL, Westcott WL. Correlates of upper and lower body muscular strength in children. J Strength Cond Res. 2008;22:1339-46.

61. Rankinen T, Zuberi A, Chagnon YC, Weisnagel SJ, Argyropoulos G, Walts B, Pérusse L, Bouchard C. The human obesity gene map: the 2005 update. Obesity. 2006;14:529-644.

62. Thorleifsson G, Walters GB, Gudbjartsson DF et al. Genome-wide association yields new sequence variants at seven loci that associate with measures of obesity. Nat Genet. 2009;41:18-24.

63. Walley AJ, Asher JE, Froguel P. The genetic contribution to nonsyndromic human obesity. Nat Rev Genet. 2009;10:431-42.

64. Bogardus C. Missing heritability and GWAS utility. Obesity. 2009;17:209-10. 
López-Jaramillo P., Cohen D., Gómez-Arbeláez D., Velandia C., Sotomayor-Rubio A., Rincón-Romero, K., López-López J.

65. Walters RG, Jacquemont S, Valsesia A et al. A new highly penetrant form of obesity due to deletions on chromosome 16p11.2. Nature. 2011;463:671-75.

66. Campion J, Milagro FI, Martinez JA. Individuality and epigenetics in obesity. Obes Rev. 2009;10:383-92.
67. Heerwagen MJ, Miller MR, Barbour LA, Friedman JE. Maternal Obesity and Fetal Metabolic Programming: A Fertile Epigenetic Soil. Am J Physiol Regul Integr Comp Physiol. 2010;299:711-22.J 